\title{
THE INDEX OF REDUCIBILITY OF PARAMETER IDEALS AND MOSTLY ZERO FINITE LOCAL COHOMOLOGIES
}

\author{
JUNG-CHEN LIU AND MARK W. ROGERS
}

\begin{abstract}
In this paper we prove that if $M$ is a finitely-generated module of dimension $d$ with finite local cohomologies over a Noetherian local ring $(A, \mathfrak{m})$, and if $\mathrm{H}_{\mathfrak{m}}^{i}(M)=0$ except possibly for $i \in\{0, r, d\}$ with some $0 \leq r \leq d$, then there exists an integer $\ell$ such that every parameter ideal for $M$ contained in $\mathfrak{m}^{\ell}$ has the same index of reducibility. This theorem generalizes earlier work of the second author and is closely related to recent work of GotoSuzuki and Goto-Sakurai; Goto-Sakurai have supplied an answer of yes in case $M$ is Buchsbaum.
\end{abstract}

\section{INTRODUCTION}

Throughout this paper, $A$ denotes a Noetherian local ring with maximal ideal $\mathfrak{m}$ and residue field $k=A / \mathfrak{m}$, and $M$ is a finitely generated $A$-module of dimension $d$. In the same 1921 paper where she introduced the fundamental notion of primary decomposition of ideals, E. Noether showed that every ideal in a Noetherian ring may be expressed as a finite intersection of irreducible ideals. She also showed that if the intersection is irredundant, then the number of irreducible ideals appearing in the intersection depends only on the ideal and not on the particular intersection. This result readily generalizes to Noetherian modules. For a submodule $N$ of $M$, we refer to the number of irreducible submodules appearing in any irredundant expression of $N$ as an intersection of irreducible submodules as the index of reducibility of $N$.

By a parameter ideal for $M$ we mean an ideal $\mathfrak{q}$ that can be generated by $d$ elements and such that $M / \mathfrak{q} M$ has finite length. We define the index of reducibility of $\mathfrak{q}$ on $M$ to be the index of reducibility of the submodule $\mathfrak{q} M$; we denote the index of reducibility of $\mathfrak{q}$ on $M$ by $\mathrm{N}_{A}(\mathfrak{q} ; M)$. Since $M / \mathfrak{q} M$ has finite length, it is known that the index of

1991 Mathematics Subject Classification. Primary 13D45; Secondary 13H10.

Key words and phrases. index of reducibility, socle, parameter ideal, type, local cohomology, finite local cohomologies, unmixed component. 
reducibility of a parameter ideal $\mathfrak{q}$ on $M$ is given by the socle dimension of $M / \mathfrak{q} M$; that is, $\mathrm{N}_{A}(\mathfrak{q} ; M)=\operatorname{dim}_{k} \operatorname{Hom}_{A}(k, M)$. (The socle of $M$, which we denote by $\operatorname{Soc}(M)$, is generally defined as the sum of all the simple submodules of $M$; in our setting, it equals $\left(0:_{M} \mathfrak{m}\right)$ and is isomorphic to $\operatorname{Hom}_{A}(k, M)$.)

In 1956, D. G. Northcott showed that the index of reducibility of a parameter ideal in a Cohen-Macaulay local ring depends only on the ring and not on the parameter ideal [N, Theorem 3]. This result extends to modules, and the common index of reducibility of parameter ideals on a Cohen-Macaulay module is called the type of the module. Regardless of whether $M$ is Cohen-Macaulay, the type is defined to be $\operatorname{dim}_{k} \operatorname{Ext}_{A}^{t}(k, M)$, where $t$ is the depth of $M$.

Although Northcott and D.G. Rees proved in 1956 that if every parameter ideal of a Noetherian local ring is irreducible then the ring is Cohen-Macaulay [NR], the constant index of reducibility of parameter ideals does not characterize Cohen-Macaulay local rings. Indeed, in 1964 S. Endo and M. Narita gave examples of non-Cohen-Macaulay Noetherian local rings having constant index of reducibility of parameter ideals EN.

In 1984, S. Goto and N. Suzuki revived interest in the index of reducibility of parameter ideals, generalizing the examples of EndoNarita, and paying particular attention to the supremum of the index of reducibility of parameter ideals GSu. We refer to this supremum as the Goto-Suzuki type. Although examples were given showing that the Goto-Suzuki type may be infinity, finite upper and lower bounds were provided in the case where $M$ has finite local cohomologies GSu, Theorem 2.1 and Theorem 2.3].

Definition 1.1. We say that $M$ has finite local cohomologies if the local cohomology $A$-modules $\mathrm{H}_{\mathfrak{m}}^{i}(M)$ have finite length for each integer $i$ such that $0 \leq i \leq d-1$.

Implicit in [GSu we find that if $M$ is a module having finite local cohomologies, there exists inside every power of $\mathfrak{m}$ a parameter ideal $\mathfrak{q}$ such that

$$
\mathrm{N}_{A}(\mathfrak{q} ; M)=\sum_{i=0}^{d}\left(\begin{array}{l}
d \\
i
\end{array}\right) \operatorname{Socdim}\left(\mathrm{H}_{\mathfrak{m}}^{i}(M)\right) .
$$

This is the lower bound for the Goto-Suzuki type mentioned above.

An equivalent condition for $M$ to have finite local cohomologies is the existence of a standard ideal for $M$.

Definition 1.2. An ideal $\mathfrak{a}$ is called a standard ideal for $M$ if $\mathfrak{a}$ is an $\mathfrak{m}$-primary ideal with the property that for each system of parameters 
$x_{1}, \ldots, x_{d}$ of $M$ contained in $\mathfrak{a}$, we have

$$
\left(\left(x_{1}, \ldots, x_{i-1}\right) M:_{M} x_{i}\right)=\left(\left(x_{1}, \ldots, x_{i-1}\right) M:_{M} \mathfrak{a}\right)
$$

for every integer $i$ with $1 \leq i \leq d$.

In 2003, Goto and H. Sakurai showed that if $M$ is Buchsbaum (i.e., $\mathfrak{m}$ is a standard ideal for $M$ ), then there exists a power of $\mathfrak{m}$ inside which every parameter ideal for $M$ has the same index of reducibility on $M$ [GSa, Corollary 3.13], necessarily equal to the lower bound of the Goto-Suzuki type. We refer to this by saying $M$ has eventual constant index of reducibility of parameter ideals. In a recent paper, the second author showed that if $M$ has dimension one, or if $M$ has dimension two, finite local cohomologies, and positive depth, then $M$ has eventual constant index of reducibility of parameter ideals $\mathbb{R}$, Theorem 2.3 and Theorem 3.3]. The results mentioned in this paragraph provide partial answers to the following question, which appears as [R, Question 1.2].

Question 1.3. Suppose $(A, \mathfrak{m}, k)$ is a Noetherian local ring having finite local cohomologies. Does A have eventual constant index of reducibility of parameter ideals?

In this paper we provide a partial answer to this question by generalizing the results in $[\mathrm{R}$. Our main result is the following theorem.

Theorem 1.4 (Main Theorem).

Suppose $M$ has finite local cohomologies and that $\mathrm{H}_{\mathfrak{m}}^{i}(M)=0$ except possibly for $i \in\{0, r, d\}$, where $r$ is some integer with $0 \leq r \leq d$. There exists an integer $\ell$ such that for every parameter ideal $\mathfrak{q}$ of $M$, if $\mathfrak{q} \subseteq \mathfrak{m}^{\ell}$ then the index of reducibility of $\mathfrak{q}$ on $M$ is independent of $\mathfrak{q}$ and is given by

$$
\mathrm{N}_{A}(\mathfrak{q} ; M)=\sum_{i=0}^{d}\left(\begin{array}{l}
d \\
i
\end{array}\right) \operatorname{Socdim}\left(\mathrm{H}_{\mathfrak{m}}^{i}(M)\right)
$$

In the process of proving this result, we provide information on the behavior of the unmixed components of parts of systems of parameters contained in a standard ideal. As a corollary to the Main Theorem, we present a result that characterizes a certain class of finite local cohomology modules as Gorenstein precisely when every power of the maximal ideal contains an irreducible parameter ideal (here we take as definition that a Gorenstein local ring is a Cohen-Macaulay local ring having an irreducible parameter ideal.) 


\section{Proof of the MAIN THEOREM AND A COROLlARY}

The importance of the existence of a standard ideal is made clear by the following proposition, for which we refer the reader to SV] Corollary 18, p. 264].

Proposition 2.1. There is a standard ideal for $M$ if and only if $M$ has finite local cohomologies.

Now we present the proof of the main theorem, Theorem 1.4, the statement and proof of several useful lemmas are postponed.

Proof. Set $W=\mathrm{H}_{\mathfrak{m}}^{0}(M)$ and $\bar{M}=M / W$. According to Proposition 3.1 there is an integer $n$ so that for every parameter ideal $\mathfrak{q}$ for $M$, if $\mathfrak{q} \subseteq \mathfrak{m}^{n}$ then $\mathrm{N}_{A}(\mathfrak{q} ; M)=\operatorname{Socdim}(M)+\mathrm{N}_{A}(\mathfrak{q} ; \bar{M})$. Since $\mathrm{H}_{\mathfrak{m}}^{i}(\bar{M}) \cong \mathrm{H}_{\mathfrak{m}}^{i}(M)$ for all $i>0$, we may require the integer $\ell$ to be at least as large as $n$ and replace $M$ by $\bar{M}$ for the remainder of the proof to assume that depth $M>0$. If $\mathrm{H}_{\mathfrak{m}}^{i}(M)=0$ for all $i<d$, then we're done since $M$ is Cohen-Macaulay. Otherwise, we have that $\mathrm{H}_{\mathfrak{m}}^{i}(M)=0$ for all $i$ except $i=d$ and $i=r$ where $0<r<d$.

For a submodule $N$ of $M$, we denote by $\mathrm{U}(N)$ the unmixed component of $N$; that is, $\mathrm{U}(N)$ is the intersection of the primary components of $N$ whose associated primes have maximal dimension, equal to $\operatorname{dim} M / N$. According to $[\mathrm{R}$, Proposition 3.2], if we let $\mathfrak{a}$ be a standard ideal for $M$ then there exists an integer $\ell$ such that $\mathfrak{m}^{\ell} \subseteq \mathfrak{a}$ and such that for any parameter ideal $\mathfrak{q}=\left(x_{1}, \ldots, x_{d}\right) A$ for $M$, if $\mathfrak{q} \subseteq \mathfrak{m}^{\ell}$ then the index of reducibility of $\mathfrak{q}$ on $M$ is given by

$$
\mathrm{N}_{A}(\mathfrak{q} ; M)=\operatorname{Socdim}\left(\sum_{i=1}^{d} \frac{U_{i}+\mathfrak{q} M}{\mathfrak{q} M}\right)+\operatorname{Socdim}\left(\mathrm{H}_{\mathfrak{m}}^{d}(M)\right)
$$

where $U_{i}=\mathrm{U}\left(\left(x_{1}, \ldots, \widehat{x_{i}}, \ldots, x_{d}\right) M\right)$. Our task in this proof is to examine the sum appearing in the expression above.

We begin by using the assumption that $\mathrm{H}_{\mathfrak{m}}^{i}(M)=0$ for all integers $i$ with $r<i<d$ to apply Proposition 3.7 and obtain

$$
\sum_{i=1}^{d} \frac{U_{i}+\mathfrak{q} M}{\mathfrak{q} M}=\sum_{1 \leq i_{1}<\cdots<i_{r} \leq d} \frac{\mathrm{U}\left(\left(x_{i_{1}}, \ldots, x_{i_{r}}\right) M\right)+\mathfrak{q} M}{\mathfrak{q} M} .
$$

Next we examine one of the summands on the right side. Using Lemma 3.8 we see that

$$
\frac{\mathrm{U}\left(\left(x_{i_{1}}, \ldots, x_{i_{r}}\right) M\right)+\mathfrak{q} M}{\mathfrak{q} M} \cong \frac{\mathrm{U}\left(\left(x_{i_{1}}, \ldots, x_{i_{r}}\right) M\right)}{\left(x_{i_{1}}, \ldots, x_{i_{r}}\right) M} .
$$


Since $\mathrm{H}_{\mathfrak{m}}^{i}(M)=0$ for each integer $i$ such that $0 \leq i \leq r-1$, we use Proposition 3.13 to obtain

$$
\frac{\mathrm{U}\left(\left(x_{i_{1}}, \ldots, x_{i_{r}}\right) M\right)+\mathfrak{q} M}{\mathfrak{q} M} \cong \mathrm{H}_{\mathfrak{m}}^{r}(M) .
$$

It remains to show that the sum

$$
\sum_{1 \leq i_{1}<\cdots<i_{r} \leq d} \frac{\mathrm{U}\left(\left(x_{i_{1}}, \ldots, x_{i_{r}}\right) M\right)+\mathfrak{q} M}{\mathfrak{q} M}
$$

is direct.

By symmetry, it suffices to show that $\mathfrak{q} M$ equals the module

$$
\left[\mathrm{U}\left(\left(x_{1}, \ldots, x_{r}\right) M\right)+\mathfrak{q} M\right] \cap\left[\sum_{\substack{1 \leq i_{1}<\cdots<i_{r} \leq d \\ i_{r}>r}} \mathrm{U}\left(\left(x_{i_{1}}, \ldots, x_{i_{r}}\right) M\right)+\mathfrak{q} M\right] .
$$

Using basic properties of intersections, this is equivalent to showing that

$$
\mathrm{U}\left(\left(x_{1}, \ldots, x_{r}\right) M\right) \cap\left[\sum_{\substack{1 \leq i_{1}<\cdots<i_{r} \leq d \\ i_{r}>r}} \mathrm{U}\left(\left(x_{i_{1}}, \ldots, x_{i_{r}}\right) M\right)\right] \subseteq \mathfrak{q} M .
$$

Using Lemma [3.4, we see that each summand $\mathrm{U}\left(\left(x_{i_{1}}, \ldots, x_{i_{r}}\right) M\right)$, where $i_{r}>r$, is contained in at least one of the submodules

$$
\mathrm{U}\left(\left(x_{1}, \ldots, \widehat{x_{i}}, \ldots, x_{d}\right) M\right)
$$

$(1 \leq i \leq r)$. Thus the intersection above is contained in

$$
\mathrm{U}\left(\left(x_{1}, \ldots, x_{r}\right) M\right) \cap\left[\sum_{i=1}^{r} \mathrm{U}\left(\left(x_{1}, \ldots, \widehat{x_{i}}, \ldots, x_{d}\right) M\right)\right] .
$$

According to Proposition 3.14, this intersection is contained in the submodule $\left(x_{1}, \ldots, x_{r}\right) M$. This completes the proof of the theorem.

Corollary 2.2. Suppose $A$ is a Noetherian local ring having finite local cohomologies and that $\mathrm{H}_{\mathfrak{m}}^{i}(A)$ is zero except possibly for $i \in\{0, r, d\}$, where $r$ is some integer with $0 \leq r \leq d$, and $d=\operatorname{dim} A$. Then $A$ is Gorenstein if and only if every power of $\mathfrak{m}$ contains an irreducible parameter ideal.

Proof. It is well known that if $A$ is Gorenstein then every parameter ideal is irreducible. Now suppose that every power of $\mathfrak{m}$ contains an irreducible parameter ideal. According to the Main Theorem, each 
parameter ideal inside a high enough power of the maximal ideal has index of reducibility

$$
\sum_{i=0}^{d}\left(\begin{array}{l}
d \\
i
\end{array}\right) \operatorname{Socdim}\left(\mathrm{H}_{\mathfrak{m}}^{i}(A)\right)
$$

this expression must equal 1. Since $\mathrm{H}_{\mathfrak{m}}^{d}(A)$ is a nonzero Artinian module, it has a nonzero socle. Thus the socles of $\mathrm{H}_{\mathfrak{m}}^{i}(A)(i<d)$ must be zero, so that the Artinian modules $\mathrm{H}_{\mathfrak{m}}^{i}(A)$ are themselves zero. Since $A$ has only one nonzero local cohomology module, $A$ is Cohen-Macaulay. Since $A$ is Cohen-Macaulay with an irreducible parameter ideal, $A$ is Gorenstein. This completes the proof of the corollary.

\section{SUPPORTING PROPOSITIONS}

This section contains the supporting propositions used in the proof of the Main Theorem.

\subsection{Reduction to positive depth.}

Proposition 3.1. Let $W=\mathrm{H}_{\mathfrak{m}}^{0}(M)$. There exists an integer $n$ such that for every parameter ideal $\mathfrak{q}$ for $M$, if $\mathfrak{q} \subseteq \mathfrak{m}^{n}$ then the index of reducibility of $\mathfrak{q}$ on $M$ is given by

$$
\mathrm{N}_{A}(\mathfrak{q} ; M)=\operatorname{Socdim}(M)+\mathrm{N}_{A}(\mathfrak{q} ; M / W) .
$$

Proof. Since $W$ has finite length, there is a positive integer $a$ such that $W \cap \mathfrak{m}^{a} M=0$. Set $\bar{M}=M / W$. Since $\bar{M}$ has positive depth, there is an $\bar{M}$-regular element $x$ of $A$. By the Artin-Rees Lemma, there is a positive integer $b$ such that for all positive integers $c$, we have $\mathfrak{m}^{b+c} \bar{M} \cap x \bar{M}=\mathfrak{m}^{c}\left(\mathfrak{m}^{b} \bar{M} \cap x \bar{M}\right)$. Let $n=a+b$ and suppose $\mathfrak{q}$ is a parameter ideal for $M$ that is contained in $\mathfrak{m}^{n}$.

Since $\mathfrak{q} M \cap W=0$, we have $(W+\mathfrak{q} M) / \mathfrak{q} M \cong W$, and thus an exact sequence

$$
0 \longrightarrow W \longrightarrow M / \mathfrak{q} M \longrightarrow \bar{M} / \mathfrak{q} \bar{M} \longrightarrow 0 .
$$

Since $\operatorname{Soc}(W)=\operatorname{Soc}(M)$, after applying the socle functor $\operatorname{Soc}(*)=$ $\left(0:_{*} \mathfrak{m}\right)$ we obtain the exact sequence

$$
0 \longrightarrow \operatorname{Soc}(M) \longrightarrow \operatorname{Soc}(M / \mathfrak{q} M) \longrightarrow \operatorname{Soc}(\bar{M} / \mathfrak{q} \bar{M}) \text {. }
$$

Using the additivity of length, we see that the proof will be complete if we show that the map $\operatorname{Soc}(M / \mathfrak{q} M) \longrightarrow \operatorname{Soc}(\bar{M} / \mathfrak{q} \bar{M})$ is surjective.

Let $s \in M$ be a representative of a nonzero element of

$$
\operatorname{Soc}(\bar{M} / \mathfrak{q} \bar{M}) \cong\left((\mathfrak{q} M+W):_{M} \mathfrak{m}\right) /(\mathfrak{q} M+W) .
$$


Let $\bar{s}$ denote the class of $s$ in $\bar{M}$. Then $\bar{s} \in(\mathfrak{q} \bar{M}: \bar{M} \mathfrak{m})$. Note that since

$x\left(\mathfrak{m}^{n} \bar{M}: \bar{M} \mathfrak{m}\right) \subseteq x\left(\mathfrak{m}^{n} \bar{M}: \bar{M} x\right)=\mathfrak{m}^{n} \bar{M} \cap x \bar{M}=\mathfrak{m}^{a}\left(\mathfrak{m}^{b} \bar{M} \cap x \bar{M}\right) \bar{M}$, so that $x\left(\mathfrak{m}^{n} \bar{M}: \bar{M} \mathfrak{m}\right) \subseteq x \mathfrak{m}^{a}$. Since $x$ is $\bar{M}$-regular, we have

$$
\left(\mathfrak{m}^{n} \bar{M}: \bar{M} \mathfrak{m}\right) \subseteq \mathfrak{m}^{a} \bar{M}
$$

Since $\mathfrak{q} \subseteq \mathfrak{m}^{n}$, we see that $\bar{s} \in \mathfrak{m}^{a} \bar{M}$, so that $s \in \mathfrak{m}^{a} M+W$. Replace $s$ by another representative of $\bar{s}$ so that we may assume $s \in \mathfrak{m}^{a} M$.

Now we have $\mathfrak{m} s \subseteq \mathfrak{m}^{a+1} M \cap(\mathfrak{q} M+W)$. Since $\mathfrak{q} M \subseteq \mathfrak{m}^{a+1} M$ and $\mathfrak{m}^{a+1} M \cap W=0$, we have $\mathfrak{m} s \subseteq \mathfrak{q} M$. Thus $s \in \operatorname{Soc}(M / \mathfrak{q} M)$. This shows that the map $\operatorname{Soc}(M / \mathfrak{q} M) \longrightarrow \operatorname{Soc}(\bar{M} / \mathfrak{q} \bar{M})$ is surjective, so our proof is complete.

\subsection{The unmixed component of a sum versus the sum of the unmixed components.}

Definition 3.2. Let $N$ be a submodule of $M$. We say that $N$ is unmixed up to $\mathfrak{m}$-primary components if $\mathfrak{p} \in$ Ass $M / N$ implies that either $\mathfrak{p}=\mathfrak{m}$ or $\operatorname{dim} A / \mathfrak{p}=\operatorname{dim} M / N$.

\section{Remark 3.3.}

(1) The unmixed component does not depend on a particular primary decomposition.

(2) If $N$ is a submodule of $M$ that is unmixed up to $\mathfrak{m}$-primary components, then $\mathrm{H}_{\mathfrak{m}}^{0}(M / N) \cong \mathrm{U}(N) / N$.

(3) According to [SV] Lemma 2.2, p. 71], if $M$ has finite local cohomologies then for any part $x_{1}, \ldots, x_{r}(r \geq 0)$ of a system of parameters for $M$, the submodule $\left(x_{1}, \ldots, x_{r}\right) M$ is unmixed up to $\mathfrak{m}$-primary components.

The following lemma explains how we usually think of the unmixed component of a submodule generated by part of a system of parameters contained in a standard ideal.

Lemma 3.4. Suppose $M$ has finite local cohomologies and let $\mathfrak{a}$ be a standard ideal for $M$. Suppose $x_{1}, \ldots, x_{r}(1 \leq r \leq d)$ is part of a system of parameters for $M$. If $\left(x_{1}, \ldots, x_{r}\right) A \subseteq \mathfrak{a}$ then

$$
\mathrm{U}\left(\left(x_{1}, \ldots, x_{r-1}\right) M\right)=\left(\left(x_{1}, \ldots, x_{r-1}\right) M:_{M} x_{r}\right) .
$$

Proof. Since $\left(x_{1}, \ldots, x_{r-1}\right) M$ is unmixed up to $\mathfrak{m}$-primary components, there is an integer $n$ such that

$$
\mathrm{U}\left(\left(x_{1}, \ldots, x_{r-1}\right) M\right)=\left(\left(x_{1}, \ldots, x_{r-1}\right) M:_{M} x_{r}^{n}\right) .
$$


This last expression equals $\left(\left(x_{1}, \ldots, x_{r-1}\right) M:_{M} x_{r}\right)$, since they are both equal to $\left(\left(x_{1}, \ldots, x_{r-1}\right) M:_{M} \mathfrak{a}\right)$.

The following lemma is essentially a collection of several results from SV].

Lemma 3.5. Suppose $M$ has finite local cohomologies and let $\mathfrak{a}$ be a standard ideal with respect to $M$. Suppose $x_{1}, \ldots, x_{r}(1 \leq r \leq d)$ is part of a system of parameters for $M$. If $\left(x_{1}, \ldots, x_{r}\right) A \subseteq \mathfrak{a}$, then for all integers $n_{1}, \ldots, n_{r} \geq 1$ we have

$$
\begin{aligned}
& \left(\left(x_{1}^{n_{1}+1}, \ldots, x_{r}^{n_{r}+1}\right) M:_{M} x_{1}^{n_{1}} \cdots x_{r}^{n_{r}}\right) \\
& \quad=\left(x_{1}, \ldots, x_{r}\right) M+\sum_{i=1}^{r} \mathrm{U}\left(\left(x_{1}, \ldots, \widehat{x_{i}}, \ldots, x_{r}\right) M\right) .
\end{aligned}
$$

Proof. According to SV, Theorem 20, Lemma 23, Lemma 24, pp 264266], we have

$$
\begin{aligned}
& \left(\left(x_{1}^{n_{1}+1}, \ldots, x_{r}^{n_{r}+1}\right) M:_{M} x_{1}^{n_{1}} \cdots x_{r}^{n_{r}}\right) \\
& \quad=\left(x_{1}, \ldots, x_{r}\right) M+\sum_{i=1}^{r}\left(\left(x_{1}, \ldots, \widehat{x_{i}}, \ldots, x_{r}\right) M:_{M} x_{i}\right) .
\end{aligned}
$$

We complete the proof with an application of Lemma 3.4 .

Now we recall the connection between Koszul cohomology and local cohomology. Let $I$ be an ideal generated by elements $y_{1}, \ldots, y_{r}$. For a positive integer $n$, we use $\underline{y}^{n}$ to denote the sequence $y_{1}^{n}, \ldots, y_{r}^{n}$. We use $\mathrm{H}^{i}(\underline{y} ; M)$ to denote the $i$ th cohomology module of the Koszul cocomplex $\overline{\mathrm{K}}^{\bullet}(\underline{y} ; M)$. Since there are containments of ideals

$$
(\underline{y}) A \supseteq\left(\underline{y}^{2}\right) A \supseteq\left(\underline{y}^{3}\right) A \supseteq \cdots,
$$

for each $i$ the corresponding Koszul cohomology modules fit into a direct system

$$
\mathrm{H}^{i}(\underline{y} ; M) \longrightarrow \mathrm{H}^{i}\left(\underline{y}^{2} ; M\right) \longrightarrow \mathrm{H}^{i}\left(\underline{y}^{3} ; M\right) \longrightarrow \cdots .
$$

When $i=r$, the differentials are particularly simple: they are each multiplication by the product $y_{1} \cdots y_{r}$.

For each $i$, it is known that the direct limit of the Koszul cohomology is isomorphic to the cohomology module $\mathrm{H}_{I}^{i}(M)$; that is,

$$
\underset{n}{\lim } \mathrm{H}^{i}\left(\underline{y}^{n} ; M\right) \cong \mathrm{H}_{I}^{i}(M) \text {. }
$$


Lemma 3.6. Suppose $M$ has finite local cohomologies and $\mathrm{H}_{\mathfrak{m}}^{r}(M)=0$ for some $1 \leq r \leq d-1$. If $x_{1}, \ldots, x_{r+1}$ is part of a system of parameters for $M$, then there exists an integer $n$ such that

$$
\left(\left(x_{1}, \ldots, x_{r}\right) M:_{M} x_{r+1}\right) \subseteq\left(\left(x_{1}^{n+1}, \ldots, x_{r}^{n+1}\right) M:_{M}\left(x_{1} \cdots x_{r}\right)^{n}\right) .
$$

Proof. Let $\mathfrak{q}_{r}=\left(x_{1}, \ldots, x_{r}\right) A$ and $\mathfrak{q}_{r+1}=\left(x_{1}, \ldots, x_{r+1}\right) A$. According to [SV, Lemma 1.5, p. 28], we have the following commutative diagram:

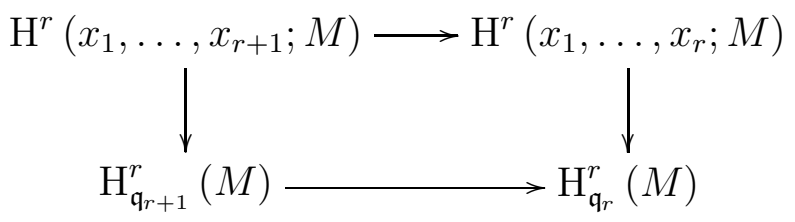

According to [SV] Lemma 22, p. 264], we have $\mathrm{H}_{\mathfrak{q}_{r+1}}^{r}(M) \cong \mathrm{H}_{\mathfrak{m}}^{r}(M)$, and by hypothesis, this module is zero. (The application of this lemma is the only place in the proof that we need finite local cohomologies.) Thus, our diagram becomes:

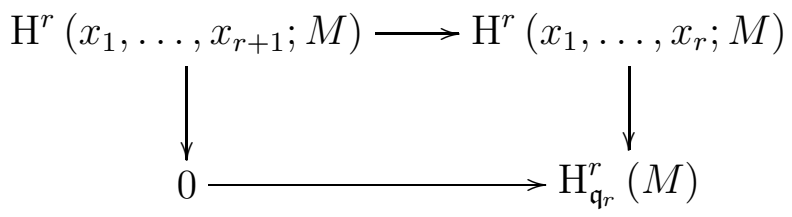

Now, we apply the covariant functor $\mathrm{H}^{0}\left(x_{r+1} ;-\right)$, which simply returns the annihilator of $x_{r+1}$ :

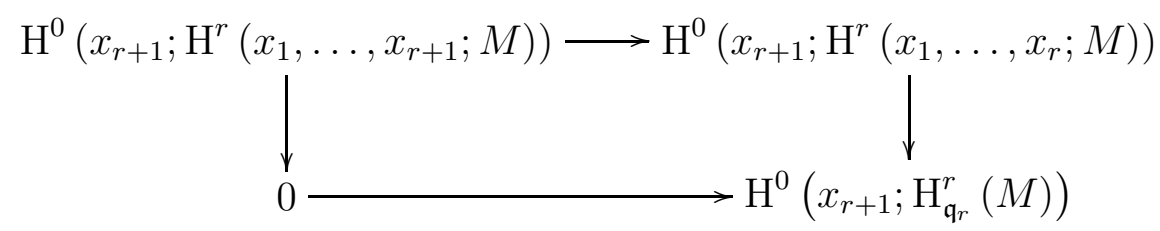

Since $x_{r+1}$ annihilates $\mathrm{H}^{r}\left(x_{1}, \ldots, x_{r+1} ; M\right)$, we have

$$
\mathrm{H}^{0}\left(x_{r+1} ; \mathrm{H}^{r}\left(x_{1}, \ldots, x_{r+1} ; M\right)\right)=\mathrm{H}^{r}\left(x_{1}, \ldots, x_{r+1} ; M\right) .
$$

From [SV, Corollary 1.7, p. 29] we know that the top row in our diagram has become surjective. Thus we have the following commutative diagram:

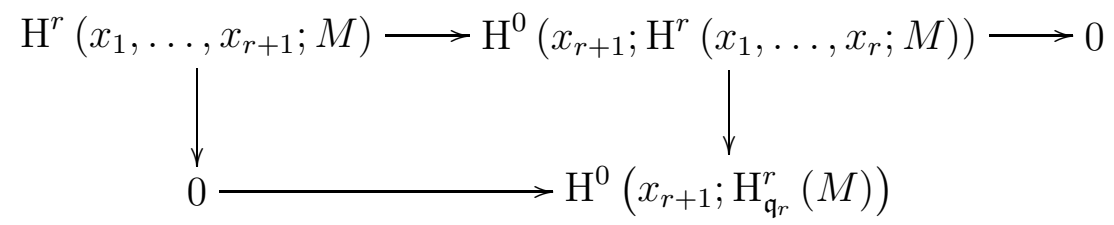


From a simple diagram chase, we see that the map

$$
\mathrm{H}^{0}\left(x_{r+1} ; \mathrm{H}^{r}\left(x_{1}, \ldots, x_{r} ; M\right)\right) \longrightarrow \mathrm{H}^{0}\left(x_{r+1} ; \mathrm{H}_{\mathfrak{q}_{r}}^{r}(M)\right)
$$

is the zero map. Thus the submodule

$$
\mathrm{H}^{0}\left(x_{r+1} ; \mathrm{H}^{r}\left(x_{1}, \ldots, x_{r} ; M\right)\right) \subseteq \mathrm{H}^{r}\left(x_{1}, \ldots, x_{r} ; M\right)
$$

is contained in the kernel $K$ of the canonical map (obtained from the direct limit) $\mathrm{H}^{r}\left(x_{1}, \ldots, x_{r} ; M\right) \longrightarrow \mathrm{H}_{\mathfrak{q}_{r}}^{r}(M)$. Recall that the cohomology module $\mathrm{H}^{r}\left(x_{1}, \ldots, x_{r} ; M\right)$ is the module $M / \mathfrak{q}_{r} M$. It follows from the definition of the direct limit that

$$
K=\frac{\cup_{n \geq 1}\left(\left(x_{1}^{n+1}, \ldots, x_{r}^{n+1}\right) M:_{M}\left(x_{1} \cdots \cdots x_{r}\right)^{n}\right)}{\mathfrak{q}_{r} M} .
$$

Furthermore,

$$
\mathrm{H}^{0}\left(x_{r+1} ; \mathrm{H}^{r}\left(x_{1}, \ldots, x_{r} ; M\right)\right)=\frac{\left(\mathfrak{q}_{r} M:_{M} x_{r+1}\right)}{\mathfrak{q}_{r} M} .
$$

Thus we have shown that

$$
\frac{\left(\mathfrak{q}_{r} M:_{M} x_{r+1}\right)}{\mathfrak{q}_{r} M} \subseteq \frac{\cup_{n \geq 1}\left(\left(x_{1}^{n+1}, \ldots, x_{r}^{n+1}\right) M:_{M}\left(x_{1} \cdots x_{r}\right)^{n}\right)}{\mathfrak{q}_{r} M} .
$$

Since the union on the right side is a union of increasing submodules, there exists an integer $n$ so that

$$
\frac{\left(\mathfrak{q}_{r} M:_{M} x_{r+1}\right)}{\mathfrak{q}_{r} M} \subseteq \frac{\left(\left(x_{1}^{n+1}, \ldots, x_{r}^{n+1}\right) M:_{M}\left(x_{1} \cdots \cdots x_{r}\right)^{n}\right)}{\mathfrak{q}_{r} M} .
$$

Thus our proof is complete.

Proposition 3.7. Suppose that $M$ has finite local cohomologies and let $\mathfrak{a}$ be a standard ideal for $M$. Suppose that $\mathrm{H}_{\mathfrak{m}}^{r}(M)=0$ for some integer $r$ with $1 \leq r \leq d-1$. For any part of a system of parameters $x_{1}, \ldots, x_{r}$ of $M$, if $\left(x_{1}, \ldots, x_{r}\right) A \subseteq \mathfrak{a}$, then

$$
\mathrm{U}\left(\left(x_{1}, \ldots, x_{r}\right) M\right)=\left(x_{1}, \ldots, x_{r}\right) M+\sum_{i=1}^{r} \mathrm{U}\left(\left(x_{1}, \ldots, \widehat{x}_{i}, \ldots, x_{r}\right) M\right) .
$$

Proof. Let $x_{1}, \ldots, x_{r}$ be as in the statement of the proposition. For each integer $i$ with $1 \leq i \leq r$, set $\mathfrak{q}_{i}=\left(x_{1}, \ldots, \widehat{x_{i}}, \ldots, x_{r}\right) A$, where $\mathfrak{q}_{i}=0$ if $r=1$. Set $\mathfrak{q}=\left(x_{1}, \ldots, x_{r}\right) A$. Choose an element $x_{r+1}$ in $\mathfrak{a}$ so that $x_{1}, \ldots, x_{r+1}$ is again part of a system of parameters for $M$. According to Lemma 3.5. for any integer $n>0$ we have

$$
\left(\left(x_{1}^{n+1}, \ldots, x_{r}^{n+1}\right) M:_{M}\left(x_{1} \cdots x_{r}\right)^{n}\right)=\mathfrak{q} M+\sum_{i=1}^{r} \mathrm{U}\left(\mathfrak{q}_{i} M\right) .
$$


From this and Lemma 3.6. it follows that

$$
\left(\mathfrak{q} M:_{M} x_{r+1}\right) \subseteq \mathfrak{q} M+\sum_{i=1}^{r} \mathrm{U}\left(\mathfrak{q}_{i} M\right) .
$$

From Lemma 3.4, we have that

$$
\mathrm{U}(\mathfrak{q} M) \subseteq \mathfrak{q} M+\sum_{i=1}^{r} \mathrm{U}\left(\mathfrak{q}_{i} M\right) .
$$

Using Lemma 3.4 again, we see that $\mathrm{U}\left(\mathfrak{q}_{i} M\right) \subseteq \mathrm{U}(\mathfrak{q} M)$ for each integer $i$ with $1 \leq i \leq r$. This completes the proof.

\subsection{The intersection of an unmixed component with a system} of parameters. The proof of the following proposition is inspired by [K. Lemma 2.2], a similar result that is stated in terms of $d$-sequences.

Proposition 3.8. Suppose $M$ has finite local cohomologies and let $\mathfrak{a}$ be a standard ideal for $M$. Let $x_{1}, \ldots, x_{r}, \ldots, x_{r+n}(0 \leq r<d, n \geq 0)$ be part of a system of parameters for $M$. If $\left(x_{1}, \ldots, x_{r+n}\right) A \subseteq \mathfrak{a}$ then

$$
\mathrm{U}\left(\left(x_{1}, \ldots, x_{r}\right) M\right) \cap\left(x_{1}, \ldots, x_{r+n}\right) M=\left(x_{1}, \ldots, x_{r}\right) M .
$$

Proof. We go by induction on $n$. The result is trivial when $n=0$.

Suppose $n>0$. Let $a$ be in $\mathrm{U}\left(\left(x_{1}, \ldots, x_{r}\right) M\right) \cap\left(x_{1}, \ldots, x_{r+n}\right) M$. Write $a=\sum_{i=1}^{r+n} x_{i} a_{i}$ with each $a_{i}$ in $M$. Since

$$
\mathrm{U}\left(\left(x_{1}, \ldots, x_{r}\right) M\right)=\left(\left(x_{1}, \ldots, x_{r}\right) M:_{M} x_{r+n}\right),
$$

we multiply $a$ by $x_{r+n}$ and obtain

$$
\sum_{i=1}^{r+n} x_{i} x_{r+n} a_{i} \in\left(x_{1}, \ldots, x_{r}\right) M .
$$

Examining the highest term, we see that $x_{r+n}^{2} a_{r+n}$ is in the submodule $\left(x_{1}, \ldots, x_{r+n-1}\right) M$. Since $\mathfrak{a}$ is a standard ideal for $M$, this implies that

$$
x_{r+n} a_{r+n} \in\left(x_{1}, \ldots, x_{r+n-1}\right) M .
$$

Recalling our expression for $a$, we see that $a$ is in $\left(x_{1}, \ldots, x_{r+n-1}\right) M$. Thus $a$ is in $\mathrm{U}\left(\left(x_{1}, \ldots, x_{r}\right) M\right) \cap\left(x_{1}, \ldots, x_{r+n-1}\right) M$. By the induction hypothesis, we see that $a$ is in $\left(x_{1}, \ldots, x_{r}\right) M$, and the proof is complete. 


\subsection{Local cohomology as a quotient of an unmixed compo- nent.}

Lemma 3.9. Suppose $M$ has finite local cohomologies and let $\mathfrak{a}$ be a standard ideal for $M$. Set $t=\operatorname{depth} M$. If $x_{1}, \ldots, x_{t}$ is part of a system of parameters for $M$ contained in $\mathfrak{a}$, then $x_{1}, \ldots, x_{t}$ is a regular sequence on $M$.

Proof. There is nothing to show if $t=0$. Suppose $t>0$. Since $\left(0:_{M} x_{1}\right)=\left(0:_{M} \mathfrak{a}\right)$, the submodule $\left(0:_{M} x_{1}\right)$ has finite length. Since $M$ has depth $t>0$, it must be that $\left(0:_{M} x_{1}\right)=0$, because $M$ has no nonzero submodule of finite length. Hence $x_{1}$ is regular on $M$.

If $t=1$, we are done. Otherwise, we go modulo the regular element $x_{1}$ and continue as above. Specifically, since $\left(x_{1} M:_{M} x_{2}\right)=$ $\left(x_{1} M:_{M} \mathfrak{a}\right), \mathfrak{a}$ kills the module $\left(0:_{M / x_{1} M} x_{2}\right)$, whence this module has finite length. Since $M / x_{1} M$ has depth $t-1>0$, we see that $\left(0: M / x_{1} M x_{2}\right)=0$, hence $x_{2}$ is regular on $M / x_{1} M$. We continue in this manner to complete the proof.

Remark 3.10. Suppose $M$ has finite local cohomologies and let $\mathfrak{a}$ be a standard ideal for $M$. Let $x_{1}, \ldots, x_{d}$ be a system of parameters for $M$ and let $\mathfrak{q}$ denote the ideal they generate. According to $[\mathrm{SV}$, Theorem and Definition 17, p. 261], if $\mathfrak{q} \subseteq \mathfrak{a}$, then we have

$$
\mathfrak{q} \mathrm{H}_{\mathfrak{m}}^{i}\left(M /\left(x_{1}, \ldots, x_{j}\right) M\right)=0
$$

for all $i$ and $j$ with $j \geq 0$ and $0 \leq i<d-j$.

Lemma 3.11. Suppose $x$ is an element of $A$ that is regular on $M$. If $x$ annihilates $\mathrm{H}_{\mathfrak{m}}^{r}(M)$ and if $\mathrm{H}_{\mathfrak{m}}^{r-1}(M)=0$, then

$$
\mathrm{H}_{\mathfrak{m}}^{r}(M) \cong \mathrm{H}_{\mathfrak{m}}^{r-1}(M / x M) .
$$

Proof. From the short exact sequence

$$
0 \longrightarrow M \longrightarrow M \longrightarrow M / x M \longrightarrow 0
$$

induced by multiplication by $x$ on $M$, we obtain the following isomorphism from the long exact sequence for local cohomology:

$$
0 \longrightarrow \mathrm{H}_{\mathfrak{m}}^{r-1}(M / x M) \longrightarrow \mathrm{H}_{\mathfrak{m}}^{r}(M) \longrightarrow 0 .
$$

The zero on the left side comes from the fact that $\mathrm{H}_{\mathfrak{m}}^{r-1}(M)=0$; the zero on the right side is due to the fact that $x \mathrm{H}_{\mathfrak{m}}^{r}(M)=0$.

Lemma 3.12. If $x_{1}, \ldots, x_{r}$ is a regular sequence on $M$ such that $\left(x_{1}, \ldots, x_{r}\right)$ A annihilates $\mathrm{H}_{\mathfrak{m}}^{r}(M)$, then

$$
\mathrm{H}_{\mathfrak{m}}^{r}(M) \cong \mathrm{H}_{\mathfrak{m}}^{0}\left(M /\left(x_{1}, \ldots, x_{r}\right) M\right) .
$$


Proof. We go by induction on $r$. If $r=1$ then we are done by Lemma 3.11.

Now assume $r>1$. By Lemma 3.11, we have an isomorphism $\mathrm{H}_{\mathfrak{m}}^{r}(M) \cong \mathrm{H}_{\mathfrak{m}}^{r-1}\left(M / x_{1} M\right)$. Set $N=M / x_{1} M$. By the induction hypothesis applied to $N$ and the sequence $x_{2}, \ldots, x_{r}$, we have $\mathrm{H}_{\mathfrak{m}}^{r-1}(N) \cong$ $\mathrm{H}_{\mathfrak{m}}^{0}\left(N /\left(x_{2}, \ldots, x_{r}\right) N\right)$. We complete the proof using this and the previous isomorphism.

Proposition 3.13. Suppose $M$ has finite local cohomologies and let $\mathfrak{a}$ be a standard ideal for $M$. Let $x_{1}, \ldots, x_{r}(0 \leq r \leq d-1)$ be part of a system of parameters for $M$ and suppose depth $M \geq r$. If $\left(x_{1}, \ldots, x_{r}\right) A \subseteq \mathfrak{a}$ then

$$
\mathrm{H}_{\mathfrak{m}}^{r}(M) \cong \mathrm{U}\left(\left(x_{1}, \ldots, x_{r}\right) M\right) /\left(x_{1}, \ldots, x_{r}\right) M .
$$

Proof. By Lemma 3.9. $x_{1}, \ldots, x_{r}$ forms a regular sequence on $M$. By Remark 3.10 we have $\left(x_{1}, \ldots, x_{r}\right) \mathrm{H}_{\mathfrak{m}}^{j}(M)=0$ for all $j$ such that $0 \leq j<d$, so from Lemma 3.12 we obtain the isomorphism $\mathrm{H}_{\mathfrak{m}}^{r}(M) \cong$ $\mathrm{H}_{\mathfrak{m}}^{0}\left(M /\left(x_{1}, \ldots, x_{r}\right) M\right)$. We complete the proof with an application of Remark 3.3

3.5. Towards the directness of a sum. This subsection is dedicated to the proof of the following proposition.

Proposition 3.14. Suppose $M$ has finite local cohomologies and let $\mathfrak{a}$ be a standard ideal for $M$. Let $x_{1}, \ldots, x_{d}$ be a system of parameters for $M$ and suppose $\left(x_{1}, \ldots, x_{d}\right) A \subseteq \mathfrak{a}$. If $M$ has positive depth and $r$ is an integer with $1 \leq r \leq \operatorname{depth} M$, then

$\mathrm{U}\left(\left(x_{1}, \ldots, x_{r}\right) M\right) \cap \sum_{i=1}^{r} \mathrm{U}\left(\left(x_{1}, \ldots, \widehat{x}_{i}, \ldots, x_{d}\right) M\right) \subseteq\left(x_{1}, \ldots, x_{r}\right) M$.

Proof. Let

$$
N=\mathrm{U}\left(\left(x_{1}, \ldots, x_{r}\right) M\right) \cap \sum_{i=1}^{r} \mathrm{U}\left(\left(x_{1}, \ldots, \widehat{x}_{i}, \ldots, x_{d}\right) M\right) .
$$

Since $\mathrm{U}\left(\left(x_{1}, \ldots, x_{r}\right) M\right)=\left(\left(x_{1}, \ldots, x_{r}\right) M:_{M} x_{d}\right)$, we see that the submodule $x_{1} \cdots x_{r} x_{d} N$ is contained in

$$
x_{1} \cdots x_{r}\left(x_{1}, \ldots, x_{r}\right) M \cap \sum_{i=1}^{r} x_{1} \cdots \widehat{x}_{i} \cdots x_{r} x_{d}\left(x_{1}, \ldots, \widehat{x}_{i}, \ldots, x_{d}\right) M .
$$


By considering $x_{d} M$ separately, we see that the previous expression equals

$$
\begin{aligned}
x_{1} \cdots x_{r}\left(x_{1}, \ldots, x_{r}\right) M \cap & {\left[\sum_{i=1}^{r} x_{1} \cdots \widehat{x}_{i} \cdots x_{r} x_{d}\left(x_{1}, \ldots, \widehat{x}_{i}, \ldots, x_{d-1}\right) M\right.} \\
& \left.+\sum_{i=1}^{r} x_{1} \cdots \widehat{x}_{i} \cdots x_{r} x_{d}^{2} M\right] .
\end{aligned}
$$

We enlarge the product on the left and see that the last expression is contained in

$$
\begin{aligned}
& \left(x_{1}^{2}, \ldots, x_{r}^{2}, x_{r+1}, \ldots, x_{d-1}\right) M \\
& \cap\left[x_{d} \sum_{i=1}^{r} x_{1} \cdots \widehat{x}_{i} \cdots x_{r}\left(x_{1}, \ldots, \widehat{x_{i}}, \ldots, x_{d-1}\right) M\right. \\
& \left.\quad+x_{d}^{2} \sum_{i=1}^{r} x_{1} \cdots \widehat{x}_{i} \cdots x_{r} M\right] .
\end{aligned}
$$

Since the first summand in the right side of the intersection is contained in the left side of the intersection, the previous expression equals

$$
\begin{aligned}
& x_{d} \sum_{i=1}^{r} x_{1} \cdots \widehat{x}_{i} \cdots x_{r}\left(x_{1}, \ldots, \widehat{x}_{i}, \ldots, x_{d-1}\right) M \\
& \quad+\left(x_{1}^{2}, \ldots, x_{r}^{2}, x_{r+1}, \ldots, x_{d-1}\right) M \cap x_{d}^{2} \sum_{i=1}^{r} x_{1} \cdots \widehat{x}_{i} \cdots x_{r} M .
\end{aligned}
$$

Since $\mathfrak{a}$ is a standard ideal, anything multiplying $x_{d}^{2}$ into the submodule

$$
\left(x_{1}^{2}, \ldots, x_{r}^{2}, x_{r+1}, \ldots, x_{d-1}\right) M
$$

also multiplies $x_{d}$ into this submodule. (We use this sort of maneuver several more times in the course of this proof.) Thus expression (3.1), and hence $x_{1} \cdots x_{r} x_{d} N$, is contained in

$$
x_{d}\left(x_{1}^{2}, \ldots, x_{r}^{2}, x_{r+1}, \ldots, x_{d-1}\right) M .
$$

Since the depth of $M$ is positive and $x_{d}$ is part of a system of parameters for $M$ that is contained in $\mathfrak{a}, x_{d}$ is regular on $M$. Hence

$$
x_{1} \cdots x_{r} N \subseteq\left(x_{1}^{2}, \ldots, x_{r}^{2}, x_{r+1}, \ldots, x_{d-1}\right) M .
$$

Now we use a similar technique that is somewhat simpler. We have that the submodule $x_{1} \cdots x_{r} x_{d-1} N$ is contained in

$$
x_{1} \cdots x_{r}\left(x_{1}, \ldots, x_{r}\right) M \cap\left[x_{d-1}\left(x_{1}^{2}, \ldots, x_{r}^{2}, x_{r+1}, \ldots, x_{d-2}\right) M+x_{d-1}^{2} M\right]
$$


Enlarging the left side of the intersection, we obtain

$$
\begin{aligned}
\left(x_{1}^{2}, \ldots,\right. & \left.x_{r}^{2}, x_{r+1}, \ldots, x_{d-2}\right) M \\
& \cap\left[x_{d-1}\left(x_{1}^{2}, \ldots, x_{r}^{2}, x_{r+1}, \ldots, x_{d-2}\right) M+x_{d-1}^{2} M\right]
\end{aligned}
$$

This equals

$$
\begin{aligned}
x_{d-1}\left(x_{1}^{2}, \ldots,\right. & \left.x_{r}^{2}, x_{r+1}, \ldots, x_{d-2}\right) M \\
& +\left(x_{1}^{2}, \ldots, x_{r}^{2}, x_{r+1}, \ldots, x_{d-2}\right) M \cap x_{d-1}^{2} M .
\end{aligned}
$$

Using the fact that $\mathfrak{a}$ is a standard ideal (as above), we see that this last expression is just $x_{d-1}\left(x_{1}^{2}, \ldots, x_{r}^{2}, x_{r+1}, \ldots, x_{d-2}\right) M$. Since $x_{d-1}$ is regular on $M$, we now see that

$$
x_{1} \cdots x_{r} N \subseteq\left(x_{1}^{2}, \ldots, x_{r}^{2}, x_{r+1}, \ldots, x_{d-2}\right) M .
$$

Continuing with the same procedure, we eventually arrive at the situation where $x_{1} \cdots x_{r} N \subseteq\left(x_{1}^{2}, \ldots, x_{r}^{2}\right) M$. Since $M$ has depth at least $r$ and $x_{1}, \ldots, x_{r}$ is part of a system of parameters for $M$ contained in a standard ideal, $x_{1}, \ldots, x_{r}$ is actually a regular sequence on $M$ (in any order). A simple proof using this fact shows that $N \subseteq\left(x_{1}, \ldots, x_{r}\right) M$, as desired. This completes the proof.

\section{EXAMPLES}

The first subsection in this section gives examples of rings to which our main theorem applies. The second subsection gives an example to show that eventual constant index of reducibility does not imply finite local cohomologies in general.

4.1. Some Rings Having Finite Local Cohomologies. It is easy to produce examples for the Main Theorem of this paper in the case $d=1$ : Any Noetherian local ring of dimension one will do. More care is required in case $d>1$; the proposition we quote below provides us with plenty of examples.

Proposition 4.1. EG, Theorem A]

Let $k$ be an infinite field and let $R=k\left[X_{1}, \ldots, X_{n}\right]$ be the full ring of polynomials over $k$ with $n$ at least 4 . Suppose that $2 \leq t_{1}<\cdots<$ $t_{s} \leq n-2$ is a sequence of integers and that $L_{1}, \ldots, L_{s}$ are graded $R$ modules of finite length. Then there is a graded prime ideal I such that $\mathrm{H}_{\mathfrak{m}}^{i}(R / I)$ is zero unless $i=t_{1}-1, \ldots, t_{s}-1, n-2$, while $\mathrm{H}_{\mathfrak{m}}^{t_{j}-1}(R / I)$ is isomorphic to $L_{j}$ for $j=1, \ldots, s$. Moreover, if $t_{1}$ is at least 3 , then $R / I$ may also be taken to be a normal domain. 
In order to obtain examples relevant to this paper, we take $s=1$ and $t_{1}=r+1$, where $r$ is the integer mentioned in the Main Theorem. We may then choose $L_{1}$ to be any $R$-module of finite length; $L_{1}$ will be the $r$ th local cohomology module of $R / I$ and all other local cohomology modules of $R / I$ will be zero, except of course for the highest, at position $n-2$. To obtain local examples, we can localize at the maximal homogeneous ideal $\left(X_{1}, \ldots, X_{n}\right) R$.

Now we turn to a more concrete example which is suggested to us by Goto. Let $k$ be a field and let $s$ and $t$ be indeterminates. In what follows, we work with a graded ring over a field instead of a local ring; to obtain a local example, we can localize at the maximal homogeneous ideal. Set $A=k\left[s^{5}, s^{4} t, s t^{4}, t^{5}\right]$ and let $\mathfrak{m}$ denote the maximal homogeneous ideal. Then $A$ is a two-dimensional graded domain, where the grading is done by total degree. We will show that $A$ has finite local cohomologies, that $\mathfrak{m}$ does not kill the first local cohomology module of $A$, and that $A$ does not have constant index of reducibility of parameter ideals.

To see that $A$ has finite local cohomologies, according to Proposition 2.1. it suffices to produce a standard system of parameters. Let $x=s^{10}$ and $y=t^{10}$; we claim that $\{x, y\}$ is a standard system of parameters. Let $\mathfrak{q}=(x, y) A$. According to [SV], Theorem and Definition 17, p. 261], it suffices to show that each of the four sequences $\{x, y\},\left\{x^{2}, y\right\}$, $\left\{x, y^{2}\right\}$, and $\left\{x^{2}, y^{2}\right\}$ is $\mathfrak{q}$-weak. Since $x$ and $y$ are regular elements, we only need to check $\left(x A:_{A} y\right)=\left(x A:_{A} \mathfrak{q}\right),\left(x^{2} A:_{A} y\right)=\left(x^{2} A:_{A} \mathfrak{q}\right)$, $\left(x A:_{A} y^{2}\right)=\left(x A:_{A} \mathfrak{q}\right)$, and $\left(x^{2} A:_{A} y^{2}\right)=\left(x^{2} A:_{A} \mathfrak{q}\right)$. Note that these ideals are monomial ideals, so they can be computed directly. In particular, we have

$$
\begin{aligned}
& \left(x A:_{A} y^{2}\right)=\left(s^{10} A:_{A} t^{20}\right)=s^{10} A+\left(s^{13} t^{2}, s^{12} t^{3}\right) A \\
& \left(x^{2} A:_{A} y^{2}\right)=\left(s^{20} A:_{A} t^{20}\right)=s^{20} A+\left(s^{23} t^{2}, s^{22} t^{3}\right) A
\end{aligned}
$$

It is not difficult to see $s^{13} t^{2} \cdot t^{10}=s^{13} t^{12}=s^{10} \cdot s^{3} t^{12}$ and $s^{12} t^{3} \cdot t^{10}=$ $s^{12} t^{13}=s^{10} \cdot s^{2} t^{13}$ are contained in $x A=s^{10} A$, so we have $s^{13} t^{2}, s^{12} t^{3} \in$ $\left(x A:_{A} y\right)$. Thus $\left(x A:_{A} y^{2}\right)=\left(x A:_{A} y\right)=\left(x A:_{A} \mathfrak{q}\right)$. Similarly, we see that $s^{23} t^{2}$ and $s^{22} t^{3}$ are in $\left(x^{2} A:_{A} y\right)$, so $\left(x^{2} A:_{A} y^{2}\right)=\left(x^{2} A:_{A} y\right)$. Moreover, we also see that $s^{23} t^{2}$ and $s^{22} t^{3}$ are in $\left(x^{2} A:_{A} x\right)$. Thus we have $\left(x^{2} A:_{A} y^{2}\right)=\left(x^{2} A:_{A} y\right)=\left(x A:_{A} \mathfrak{q}\right)$.

Since $\{x, y\}$ is a standard system of parameters, by SV], Theorem and Definition 17, p. 261], they each kill $\mathrm{H}_{\mathfrak{m}}^{1}(A)$. Moreover, since the ideal $x A$ is unmixed up to $\mathfrak{m}$-primary components, we have $\mathrm{H}_{\mathfrak{m}}^{1}(A) \cong$ $\mathrm{U}(x A) / x A$. On the other hand, by Lemma 3.4, we see that $\mathrm{U}(x A)=$ $\left(x A:_{A} y\right)=\left(s^{10}, s^{12} t^{3}, s^{13} t^{2}\right) A$; thus $\mathrm{H}_{\mathfrak{m}}^{1}(A) \cong\left(s^{10}, s^{12} t^{3}, s^{13} t^{2}\right) A / s^{10} A$. 
From this we see that $\mathrm{H}_{\mathfrak{m}}^{1}(A)$ is not killed by $\mathfrak{m}$ but it is killed by $\mathfrak{m}^{2}$. Furthermore, we compute the socle of the first local cohomology by calculating $\left(s^{10}:_{A} \mathfrak{m}\right)=\left(s^{10}, s^{13} t^{7}, s^{17} t^{3}\right) A$. Hence the socle dimension of $\mathrm{H}_{\mathfrak{m}}^{1}(A)$ is two.

Now we use the Cech complex to see that the socle dimension of $\mathrm{H}_{\mathfrak{m}}^{2}(A)$ is four. According to the Čech complex, we have

$$
\mathrm{H}_{\mathfrak{m}}^{2}(A) \cong \frac{A\left[1 / s^{5} t^{5}\right]}{A\left[1 / s^{5}\right]+A\left[1 / t^{5}\right]}
$$

We examine the components of this expression and find that

$$
\begin{aligned}
& A\left[1 / s^{5}\right]=k[t / s]\left[s^{5}, 1 / s^{5}\right]=\sum_{n \in \mathbb{Z}} k[t / s] s^{5 n}, \\
& A\left[1 / t^{5}\right]=k[s / t]\left[t^{5}, 1 / t^{5}\right]=\sum_{n \in \mathbb{Z}} k[s / t] t^{5 n},
\end{aligned}
$$

and

$$
A\left[1 / s^{5} t^{5}\right]=\sum_{n \in \mathbb{Z}} \sum_{\substack{\alpha+\beta=5 n \\ \alpha, \beta \in \mathbb{Z}}} k s^{\alpha} t^{\beta} .
$$

Notice that $1 / s t^{4}, 1 / s^{2} t^{3}, 1 / s^{3} t^{2}$, and $1 / s^{4} t$ are linearly independent and contained in the socle. Now we just need to show that nothing outside of their span is in the socle. To see this, suppose $\alpha+\beta \geq 10$ and $\alpha, \beta \geq 1$. We show that $1 / s^{\alpha} t^{\beta}$ is not in the socle. It must be true that either $\alpha-4>0$ or $\beta-4>0$. By symmetry, we may assume without loss of generality that $\alpha-4>0$. If $\beta=1$, then $\alpha \geq 9$, and the element $s^{5}\left(1 / s^{\alpha} t^{\beta}\right)=1 / s^{\alpha-5} t$ is not in $A\left[1 / s^{5}\right]+A\left[1 / t^{5}\right]$, since its denominator is not a pure power of $s$ or $t$. Thus we see that when $\beta=1$, the element $1 / s^{\alpha} t^{\beta}$ is not in the socle. If $\beta>1$, then $s^{4} t\left(1 / s^{\alpha} t^{\beta}\right)=1 / s^{\alpha-4} t^{\beta-1}$ is not in $A\left[1 / s^{5}\right]+A\left[1 / t^{5}\right]$, and we see that $1 / s^{\alpha} t^{\beta}$ is not in the socle. Hence Socdim $\left(\mathrm{H}_{\mathfrak{m}}^{2}(A)\right)=4$.

According to the Main Theorem, the index of reducibility of parameter ideals for $A$ contained in high powers of the maximal ideal is $2 \times 2+4=8$. Using the fact that the index of reducibility of a parameter ideal $\mathfrak{q}$ equals the socle dimension of $A / \mathfrak{q}$, we find that $\mathrm{N}_{A}\left(\left(s^{5}, t^{5}\right) A ; A\right)=2, \mathrm{~N}_{A}\left(\left(s^{10}, t^{10}\right) A ; A\right)=4$, and $\mathrm{N}_{A}\left(\left(s^{15}, t^{15}\right) A ; A\right)=$ 8 , thus $A$ does not have constant index of reducibility. More precisely, 
with direct computation, we have

$$
\begin{aligned}
& \left(\left(s^{5}, t^{5}\right) A:_{A} \mathfrak{m}\right)=\left(s^{5}, t^{5}, s^{12} t^{3}, s^{3} t^{12}\right) A \\
& \left(\left(s^{10}, t^{10}\right) A:_{A} \mathfrak{m}\right)=\left(s^{10}, t^{10}, s^{17} t^{3}, s^{13} t^{7}, s^{7} t^{13}, s^{3} t^{17}\right) A \\
& \left(\left(s^{15}, t^{15}\right) A:_{A} \mathfrak{m}\right)=\left(s^{15}, t^{15}, s^{22} t^{3}, s^{18} t^{7}, s^{14} t^{11}, s^{13} t^{12}\right. \\
& \left.s^{12} t^{13}, s^{11} t^{14}, s^{7} t^{18}, s^{3} t^{22}\right) A
\end{aligned}
$$

SO

$$
\begin{aligned}
& \mathrm{N}_{A}\left(\left(s^{5}, t^{5}\right) A ; A\right)=\operatorname{Socdim}\left(A /\left(s^{5}, t^{5}\right) A\right)=2 \\
& \mathrm{~N}_{A}\left(\left(s^{10}, t^{10}\right) A ; A\right)=\operatorname{Socdim}\left(A /\left(s^{10}, t^{10}\right) A\right)=4 \\
& \mathrm{~N}_{A}\left(\left(s^{15}, t^{15}\right) A ; A\right)=\operatorname{Socdim}\left(A /\left(s^{15}, t^{15}\right) A\right)=8
\end{aligned}
$$

We can also confirm certain assertions in this example by using the computer program Macaulay 2 [M].

Remark 4.2. The preceding example shows that parameter ideals contained in a standard ideal need not have the same index of reducibility. Indeed, $\left(s^{10}, t^{10}\right) A$ and $\left(s^{15}, t^{15}\right) A$ are both contained in the standard ideal $\left(s^{10}, t^{10}\right) A$, but they have different indexes of reducibility.

4.2. Eventual Constancy Does Not Imply Finite Local Cohomologies. Eventual constant index of reducibility does not imply finite local cohomologies. In fact, even constant index of reducibility does not imply finite local cohomologies, as one can see from GSu, Example 4.7]. In this section we provide another example by slightly generalizing and reexamining an example from Section 5 of [GSa].

Example 4.3. Let $n \geq 2$ and let $S$ be an $n+1$-dimensional regular local ring whose maximal ideal is $\left(x, y_{1}, y_{2}, \ldots, y_{n}\right) S$. Let $A=$ $S /\left(x y_{1}, x y_{2}, \ldots, x y_{n}\right) S$ and let $\mathfrak{m}=\left(x, y_{1}, y_{2}, \ldots, y_{n}\right) A$, the maximal ideal of $A$. Then $A$ is a Noetherian local ring with dimension $n$ and depth 1 such that every parameter ideal contained in $\mathfrak{m}^{2}$ has index of reducibility 2 , and $A$ does not have finite local cohomologies.

We note that this example does not have constant index of reducibility, since the parameter ideal $\left(y_{1}-x, y_{2}, \ldots, y_{n}\right) A$ is irreducible.

Proof. Let $\mathfrak{p}=\left(y_{1}, y_{2}, \ldots, y_{n}\right) A$. The ring $A$ does not have finite local cohomologies since its minimal primes $x A$ and $\mathfrak{p}$ have different dimensions: $\operatorname{dim} A /(x)=n$ and $\operatorname{dim} A / \mathfrak{p}=1$ [SV, Proposition 16, p. 260].

Let $\mathfrak{q}$ be a parameter ideal for $A$ contained in $\mathfrak{m}^{2}$. Consider the following exact sequence of $A$-modules:

$$
0 \longrightarrow A / \mathfrak{p} \stackrel{\cdot x}{\longrightarrow} A \longrightarrow A / x A \longrightarrow 0 \text {. }
$$


Since $A / x A \cong S / x S, A / x A$ is a Cohen-Macaulay $A$-module of dimension $n$. Since $\mathfrak{q}$ is still a parameter ideal for $A / x A$, it is generated by an $A / x A$-regular sequence, so $\operatorname{Tor}_{A}^{1}(A / \mathfrak{q}, A / x A)=0$. We tensor with $A / \mathfrak{q}$ and use fundamental isomorphisms to obtain an exact sequence

$$
0 \longrightarrow A /(\mathfrak{p}+\mathfrak{q}) \stackrel{\cdot x}{\longrightarrow} A / \mathfrak{q} \longrightarrow A /(x A+\mathfrak{q}) \longrightarrow 0 \text {. }
$$

Now we apply the socle functor:

$$
0 \longrightarrow \operatorname{Soc}(A /(\mathfrak{p}+\mathfrak{q})) \stackrel{\cdot x}{\longrightarrow} \operatorname{Soc}(A / \mathfrak{q}) \longrightarrow \operatorname{Soc}(A /(x A+\mathfrak{q})) .
$$

Since $A / \mathfrak{p}$ is a DVR and $A /(\mathfrak{p}+\mathfrak{q})$ has finite length, it follows that $\operatorname{Socdim}(A /(\mathfrak{p}+\mathfrak{q}))=1$. Since $A / x A$ is a type 1 Cohen-Macaulay $A$ module and $\mathfrak{q}$ is a parameter ideal for $A / x A, \operatorname{Socdim}(A /(x A+\mathfrak{q}))=1$. Thus, as soon as we show the map $A / \mathfrak{q} \longrightarrow A /(x A+\mathfrak{q})$ is surjective on the socles, we will have $\mathrm{N}_{A}(\mathfrak{q} ; A)=1+1=2$, and our proof will be complete.

Write $\mathfrak{q}=\left(f_{1}, f_{2}, \ldots, f_{n}\right) A$. We will modify these elements $f_{i}$ during the course of the proof. Use $₹$ to denote reduction modulo $\mathfrak{p}$. Since $\bar{A}$ is a DVR, we know that one of the ideals $\overline{f_{i} A}$ contains the others. Without loss of generality, we assume it is $\overline{f_{1} A}$. Thus there are elements $u_{i} \in A$ such that $\overline{f_{i}}=\overline{u_{i} f_{1}}$ for $2 \leq i \leq n$. Hence $f_{i}-u_{i} f_{1} \in \mathfrak{p}$ for each $2 \leq i \leq n$. Since $\mathfrak{q}=\left(f_{1}, f_{2}-u_{2} f_{1}, \ldots, f_{n}-u_{n} f_{1}\right) A$, we may replace $f_{i}$ by $f_{i}-u_{i} f_{1}$ for $2 \leq i \leq n$ to arrive at a situation where $\mathfrak{q}=\left(f_{1}, f_{2}, \ldots, f_{n}\right) A$ with each of $f_{2}, \ldots, f_{n}$ in $\mathfrak{p}$.

Since $\mathfrak{q}$ is not contained in $\mathfrak{p}$, we know that $f_{1}$ is not in $\mathfrak{p}$. Since $\mathfrak{m}=x A+\mathfrak{p}$, we may write $f_{1}=\epsilon x^{m}+g$ with $\epsilon$ a unit in $A$ and $g \in \mathfrak{p}$. Thus $\mathfrak{q}=\left(x^{m}+\epsilon^{-1} g, f_{2}, \ldots, f_{n}\right) A$. We replace $f_{1}$ by $\epsilon^{-1} g$ to arrive at a situation where $\mathfrak{q}=\left(x^{m}+f_{1}, f_{2}, \ldots, f_{n}\right) A$ with each $f_{i}$ in $\mathfrak{p}$. Furthermore, since $\mathfrak{q} \subseteq \mathfrak{m}^{2}$, we know that $m \geq 2$ and each $f_{i}$ is in $\mathfrak{m}^{2}$.

Let $a$ be an element of $A$ that maps to a socle generator for $A /(x A+$ $\mathfrak{q})$; thus $a \mathfrak{m} \subseteq x A+\mathfrak{q}$. Our goal is to show that $a \mathfrak{m} \subseteq \mathfrak{q}$. Since the image of $\mathfrak{m}$ and $\mathfrak{p}$ are the same in $A / x A$, we may assume $a \in \mathfrak{p}$. Since $x A \cap \mathfrak{p}=0, a x=0$. It remains to see that $a y_{i} \in \mathfrak{q}$ for each $1 \leq i \leq n$.

Since $a \mathfrak{m} \subseteq \mathfrak{q}+x A=\left(f_{1}, f_{2}, \ldots, f_{n}, x\right) A$, we have equations

$$
a y_{i}=u_{i 1} f_{1}+u_{i 2} f_{2}+\cdots+u_{i n} f_{n}+v_{i} x
$$

for $1 \leq i \leq n$ and elements $u_{i j}, v_{i}$ in $A$. We see that each element $v_{i} x$ is in $x A \cap \mathfrak{p}$, and is thus zero.

We may assume that each element $u_{i j}$ is either a unit or is actually in $\mathfrak{p}$. First we show that it is not possible for one of these first coefficients to be a unit; we go by way of contradiction. Suppose one of the first coefficients $u_{i 1}$ is a unit; without loss of generality, suppose it is $u_{11}$. 
Then we may solve for $f_{1}$ and write

$$
f_{1}=u_{11}^{-1} a y_{1}-u_{11}^{-1} u_{12} f_{2}-\ldots-u_{11}^{-1} u_{1 n} f_{n} .
$$

Hence

$$
\mathfrak{q}=\left(x^{m}+u_{11}^{-1} a y_{1}, f_{2}, \ldots, f_{n}\right) .
$$

Since $u_{11}^{-1} a$ maps to a socle element of $A /(x A+\mathfrak{q})$ as well, we may replace $a$ by $u_{11}^{-1} a$. Now we have $\mathfrak{q}=\left(x^{m}+a y_{1}, f_{2}, \ldots, f_{n}\right)$.

Set $R=A /\left(x, f_{2}, \ldots, f_{n}\right) A$. Then $R$ is a one-dimensional Noetherian local ring with maximal ideal $\mathfrak{n}=\mathfrak{m} R$ and a parameter ideal $Q=a y_{1} R$ such that the socle of $R / Q$ contains the image of $a$. Furthermore, since $R$ is the quotient of the regular local ring $A / x A$ by elements contained in the square of the maximal ideal, the multiplicity of $R$ is greater than 1. Set $I=\left(Q:_{R} \mathfrak{n}\right)$. Then according to [GSa, Proposition 2.3], we have that $\mathfrak{n} I=\mathfrak{n} Q$. Since $a \in I, \mathfrak{n} a \subseteq \mathfrak{n} I=\mathfrak{n} Q=\mathfrak{n} a y_{1}$. Thus $\mathfrak{n} a=\mathfrak{n} a y_{1}$, so by Nakayama's Lemma we see that $\mathfrak{n} a=0$. This is a contradiction, since $Q=a y_{1} R \subseteq \mathfrak{n} a$, and $Q$ is a parameter ideal in the one-dimensional ring $R$. Thus we see that none of the first coefficients $u_{i 1}$ can be a unit.

Now, since each of the $n$ first coefficients $u_{i 1}, 1 \leq i \leq n$, is a nonunit, we may assume they are all in $\mathfrak{p}$, and we may rewrite the equations as

$$
a y_{i}=u_{i 1}\left(x^{m}+f_{1}\right)+u_{i 2} f_{2}+\cdots+u_{i n} f_{n} .
$$

Thus we see that $a \mathfrak{m} \subseteq \mathfrak{q}$, as desired.

\section{REFERENCES}

[EG] E. Evans Jr. and P.A. Griffith, Local cohomology modules for normal domains, J. London Math. Soc., 19, (2) (1979), 277-284.

[EN] S. Endo and M. Narita, The Number of Irreducible Components of an Ideal and the Semi-Regularity of a Local Ring, Proc. Japan Acad., 40 (1964), 627-630.

[G] R. Gilmer, Commutative ring theory. In James W. Brewer and Martha K. Smith, editors, Emmy Noether: a tribute to her life and work, Pure and Applied Mathematics, pages 131-143. Marcel Dekker, Inc., New York, 1981.

[GSa] S. Goto and H. Sakurai, The equality $I^{2}=Q I$ in Buchsbaum rings, Rend. Sem. Mat. Univ. Padova, 110 (2003), 25-56.

[GSu] S. Goto and N. Suzuki, Index of Reducibility of Parameter Ideals in a Local Ring, Journal of Algebra, 87 (1984), 53-88.

[K] T. Kawasaki, On Macaulayfication of Noetherian Schemes, Trans. American Math. Soc., 352/6 (2000), 2517-2552.

$[\mathrm{M}] \quad$ D. R. Grayson and M. E. Stillman, Macaulay 2, a software system for research in algebraic geometry, Available at http://www.math.uiuc.edu/Macaulay2/

[N] D. G. Northcott, On Irreducible Ideals in Local Rings, J. London Math. Soc., 32 (1957), 82-88. 
[NR] D. G. Northcott and D. Rees, Principal Systems, Quart. J. Math., 8 (1957), 119-27.

[R] M. W. Rogers, The index of reducibility for parameter ideals in low dimension, Journal of Algebra, 278/2 (2004), 571-584

[SV] J. Stückrad and W. Vogel, Buchsbaum Rings and Applications, SpringerVerlag, Berlin, 1986

Department of Mathematics, National Taiwan Normal University, TAIPEI, TAIWAN

E-mail address: liujc@math.ntnu.edu.tw

Department of Mathematics, Southwest Missouri State University, SPRINGFIELD, MO 65805

E-mail address: markrogers@smsu.edu 